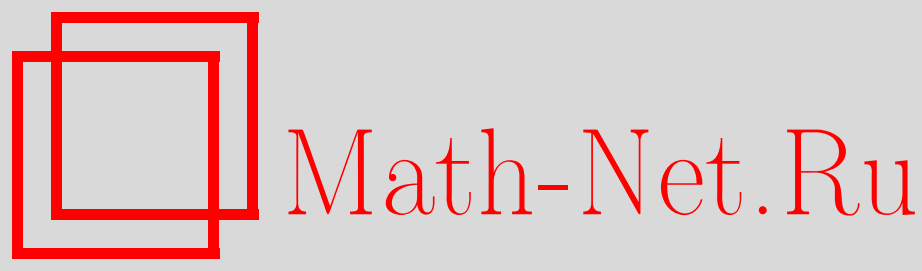

И. М. Гусейнов, А. Х. Ханмамедов, А. Ф. Мамедова, Обратная задача рассеяния для уравнения Шредингера с дополнительным квадратичным потенциалом на всей оси, ТМФ, 2018, том 195, номер 1, 54-63

DOI: https://doi.org/10.4213/tmf9423

Использование Общероссийского математического портала Math-Net.Ru подразумевает, что вы прочитали и согласны с пользовательским соглашением http://www.mathnet.ru/rus/agreement

Параметры загрузки:

IP: 54.81 .137 .203

26 апреля 2023 г., 16:50:07

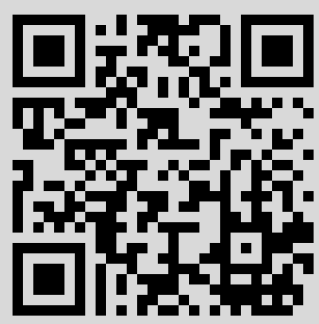




\title{
ОБРАТНАЯ ЗАДАЧА РАССЕЯНИЯ ДЛЯ УРАВНЕНИЯ ШРЕДИНГЕРА С ДОПОЛНИТЕЛЬНЫМ КВАДРАТИЧНЫМ ПОТЕНЦИАЛОМ НА ВСЕЙ ОСИ
}

\begin{abstract}
Рассмотрено уравнение Шредингера с дополнительным квадратичным потенциалом на всей оси. Методом операторов преобразования изучены прямая и обратная задачи теории рассеяния. Получены основные интегральные уравнения обратной задачи. Доказана однозначная разрешимость основных уравнений.
\end{abstract}

Ключевые слова: уравнение Шредингера, осциллятор, коэффициент отражения, обратная задача рассеяния, основные уравнения.

DOI: https://doi.org/10.4213/tmf9423

\section{1. ВВЕДЕНИЕ}

Содержательной задачей, решенной Гейзенбергом в рамках матричной механики и Шредингером на языке волновой механики, была задача о квантовом осцилляторе. K решению этой задачи сводится описание колебательных движений атомов в молекулах и кристаллах (см. [1]). Системе осцилляторов эквивалентно и "проквантованное" электромагнитное поле.

В работах [2], [3] исследовалась обратная спектральная задача, состоящая в восстановлении одномерных операторов Шредингера с дополнительным осциллятором и одинаковым дискретным спектром. Наиболее полное решение обратной задачи для возмущенного осциллятора вида $T y=-y^{\prime \prime}+x^{2} y+q(x) y$, где $q(x)$ - вещественный потенциал и $q^{\prime}(x), x q(x) \in L_{2}(-\infty,+\infty)$, дано в работе [4].

В настоящей работе изучены прямая и обратная задачи теории рассеяния для уравнения Шредингера с дополнительным квадратичным потенциалом

$$
-y^{\prime \prime}-x^{2} y+q(x) y=\lambda y, \quad-\infty<x<+\infty,
$$

* Бакинский государственный университет, Баку, Азербайджан. E-mail: agil_khanmamedov@yahoo.com

${ }^{\dagger}$ Институт математики и механики НАН Азербайджана, Баку, Азербайджан

‡Университет “Азербайджан”, Баку, Азербайджан 
где вещественный потенциал $q(x)$ является гладкой функцией и удовлетворяет условию

$$
\int_{-\infty}^{\infty}\left(1+x^{4}\right) e^{2 x^{2}}|q(x)| d x<\infty .
$$

Отметим, что при отсутствии дополнительного квадратичного потенциала, т. е. для уравнения $-y^{\prime \prime}+q(x) y=\lambda y$ с быстроубывающим потенциалом $q(x)$, обратная задача рассеяния детально изучалась в работах многих авторов (см. [5]-[7] и приведенную там литературу). Наличие дополнительного квадратичного потенциала приводит к тому, что непрерывному спектру невозмущенного уравнения отвечают собственные функции, которые, в отличие от экспоненциальной функции, не обладают мультипликативным свойством. Это обстоятельство потребовало модификации некоторых классических рассуждений из работ [5]-[7].

Заметим, что задача рассеяния для уравнения (1), заданного на полуоси, рассматривалась в работе [8]. Однако использованные в этой работе собственные функции непрерывного спектра невозмущенного уравнения не являются адекватными для уравнения (1) на всей оси ввиду появления бесконечных полюсов коэффициента прохождения. В данной работе собственные функции непрерывного спектра невозмущенного уравнения выражаются в терминах функций параболического цилиндра $D_{\nu}(z)$.

Следует отметить, что обратная задача рассеяния для одномерных уравнений Шредингера с различными растущими потенциалами изучалась в работах [9]-[13]. Вместе с тем в настоящей работе, в отличие от работ [9], [10], [12], получены оба интегральных уравнения типа Марченко, которые позволяют сделать вывод о поведении восстановленного потенциала на обоих концах.

Некоторые вопросы спектральной теории одномерного уравнения Шредингера исследовались в работах [14]-[16].

Прямая задача рассеяния для многомерного оператора Шредингера вида $H=$ $-\Delta+x_{1}^{2}+q(x), x=\left(x_{1}, x_{2}, \ldots, x_{m}\right) \in \mathbb{R}^{m}$, изучалась в работе [17].

\section{2. ПРЕДВАРИТЕЛЬНОЕ ИССЛЕДОВАНИЕ НЕВОЗМУЩЕННОГО УРАВНЕНИЯ}

Рассмотрим невозмущенное уравнение

$$
-y^{\prime \prime}-x^{2} y=\lambda y, \quad-\infty<x<+\infty .
$$

Известно (см. [18], [19]), что уравнение (3) имеет решение $\phi_{0}(x, \lambda)$, представимое в виде $\phi_{0}(x, \lambda)=D_{i \lambda / 2-1 / 2}\left(\sqrt{2} e^{i \pi / 4} x\right)$, где $U(a, x)=D_{-a-1 / 2}(x)-$ функция параболического цилиндра, она является решением уравнения

$$
-y^{\prime \prime}+\frac{x^{2}}{4} y=-a y
$$

Поведение функции $D_{\nu}(z)$ при больших значениях $|z|$ и фиксированном значении $\nu$ определяется асимптотическими формулами [19] при $z \rightarrow \infty$

$$
\begin{array}{lll}
D_{\nu}(z) \sim z^{\nu} e^{-z^{2} / 4}, & |\arg z|<\frac{3 \pi}{4}, \\
D_{\nu}(z) \sim e^{-z^{2} / 4} z^{\nu}-\frac{\sqrt{2 \pi}}{\Gamma(-\nu)} e^{i \pi \nu} e^{z^{2} / 4} z^{-\nu-1}, & \frac{\pi}{4}<\arg z<\frac{5 \pi}{4} .
\end{array}
$$


В силу последних соотношений имеем

$$
\phi_{0}(x, \lambda)=D_{i \lambda-1 / 2}\left(\sqrt{2} e^{-i \pi / 4} x\right) \sim e^{-i x^{2} / 2}\left(\sqrt{2} e^{-i \pi / 4} x\right)^{i \lambda-1 / 2}, \quad x \rightarrow+\infty .
$$

Присутствующая в правой части функция имеет порядок $O\left(x^{-(1 / 2)-\operatorname{Im} \lambda}\right)$, и, значит, $\phi_{0}(x, \lambda)$ принадлежит $L_{2}(0, \infty)$ при $\operatorname{Im} \lambda>0$. Так как уравнение $(3)$ не изменяется при замене $x$ на $-x$, то функция $\phi_{0}(-x, \lambda)$ также является его решением. Очевидно, что при $\operatorname{Im} \lambda>0$ функция $\phi_{0}(-x, \lambda)$ принадлежит $L_{2}(-\infty, 0)$.

С другой стороны, используя известные выражения [18]

$$
U\left(-\frac{i \lambda}{2}, 0\right)=\frac{2^{(i \lambda-1) / 4} \sqrt{\pi}}{\Gamma(3 / 4-i \lambda / 4)}, \quad U_{x}^{\prime}\left(-\frac{i \lambda}{2}, 0\right)=-\frac{2^{(i \lambda+1) / 4} \sqrt{\pi}}{\Gamma(1 / 4-i \lambda / 4)},
$$

находим, что при вещественных значениях $\lambda$ пара решений $\left\{\phi_{0}(x, \lambda), \overline{\phi_{0}(x, \lambda)}\right\}$ уравнения (3) линейно независимы, причем их вронскиан определяется формулой

$$
W\left\{\phi_{0}(x, \lambda), \overline{\phi_{0}(x, \lambda)}\right\}=-i \sqrt{2} e^{\pi \lambda / 4} .
$$

При вещественных значениях $\lambda$ решения $\phi_{0}( \pm x, \lambda)$ ограничены, что соответствует непрерывному спектру задачи (3). На спектре имеет место формула связи

$$
\phi_{0}(-x, \lambda)=a_{0}(\lambda) \overline{\phi_{0}(x, \lambda)}+b_{0}(\lambda) \phi_{0}(x, \lambda),
$$

причем в теории рассеяния функции $t_{0}(\lambda)=1 / a_{0}(\lambda)$ и $r_{0}(\lambda)=b_{0}(\lambda) / a_{0}(\lambda)$ имеют смысл коэффициентов прохождения и отражения соответственно. Из (5) найдем, что коэффициенты перехода $a_{0}(\lambda)$ и $b_{0}(\lambda)$ удовлетворяют условию нормировки

$$
\left|a_{0}(\lambda)\right|^{2}-\left|b_{0}(\lambda)\right|^{2}=1
$$

Более того, в силу формул (4), (5) справедливы равенства

$$
\begin{aligned}
& a_{0}(\lambda)=\frac{W\left\{\phi_{0}(x, \lambda), \phi_{0}(-x, \lambda)\right\}}{W\left\{\phi_{0}(x, \lambda), \overline{\phi_{0}(x, \lambda)}\right\}}=i \frac{\sqrt{2 \pi} e^{-i \pi / 4}}{e^{\pi \lambda / 4} \Gamma(1 / 2-i \lambda / 2)}, \\
& b_{0}(\lambda)=\frac{W\left\{\phi_{0}(-x, \lambda), \overline{\phi_{0}(x, \lambda)}\right\}}{W\left\{\phi_{0}(x, \lambda), \overline{\phi_{0}(x, \lambda)}\right\}}=i e^{-\pi \lambda / 2} .
\end{aligned}
$$

Согласно последним формулам $a_{0}(\lambda)$ и $b_{0}(\lambda)$ аналитически продолжаются в верхнюю полуплоскость $\operatorname{Im} \lambda \geqslant 0$.

Далее, следуя рассуждениям Титчмарша [20], получаем, что функции $\psi_{1}(x, \lambda)$ и $\psi_{2}(x, \lambda)$, определенные в общей теории [20] с точностью до множителя, совпадают соответственно с функциями $\phi_{0}(x, \lambda)$ и $\phi_{0}(-x, \lambda)$. Тем самым функции $\phi_{0}(x, \lambda)$, $\phi_{0}(-x, \lambda)$ служат собственными функциями непрерывного спектра уравнения (3). Следовательно, для любой функции $h(x) \in L_{2}(-\infty, \infty)$ имеют место равенства

$$
h(x)=\int_{-\infty}^{\infty} h(y)\left\{\frac{1}{\sqrt{2} \pi} \int_{-\infty}^{\infty}\left[\overline{\phi_{0}( \pm x, \lambda)}+r_{0}(\lambda) \phi_{0}( \pm x, \lambda)\right] \phi_{0}( \pm y, \lambda) \omega_{0}(\lambda) d \lambda\right\} d y
$$


где $\omega_{0}(\lambda)=e^{-\pi \lambda / 4}$. В частности, когда $h(x)$ принимает вещественные значения, последние равенства примут вид

$h(x)=\int_{-\infty}^{\infty} h(y)\left\{\frac{1}{\sqrt{2} \pi} \int_{-\infty}^{\infty} \operatorname{Re}\left\{\left[\overline{\phi_{0}( \pm x, \lambda)}+r_{0}(\lambda) \phi_{0}( \pm x, \lambda)\right] \phi_{0}( \pm y, \lambda)\right\} \omega_{0}(\lambda) d \lambda\right\} d y$.

Таким образом, справедливы следующие формулы разложения для собственных функций непрерывного спектра уравнения (3):

$$
\frac{1}{\sqrt{2} \pi} \int_{-\infty}^{\infty} \operatorname{Re}\left\{\left[\overline{\phi_{0}( \pm x, \lambda)}+r_{0}(\lambda) \phi_{0}( \pm x, \lambda)\right] \phi_{0}( \pm y, \lambda)\right\} \omega_{0}(\lambda) d \lambda=\delta(x-y),
$$

где $\delta$ - дельта-функция Дирака.

\section{3. ПРЯМАЯ ЗАДАЧА РАССЕЯНИЯ}

Перейдем к описанию задачи рассеяния уравнения (1). Задачу на собственные значения уравнения (1) будем рассматривать в классе ограниченных на всей оси функций $y=y(x)$. Вещественным значениям энергии $\lambda$ соответствует непрерывный спектр задачи (1). Собственные функции непрерывного спектра определяются асимптотическими условиями на $\pm \infty$ по $x$ :

$$
f_{ \pm}(x, \lambda)=\phi_{0}( \pm x, \lambda)+o(1), \quad x \rightarrow \pm \infty .
$$

Как следует из работы [8], для решений $f_{ \pm}(x, \lambda)$ справедливы следующие треугольные представления, демонстрирующие эффект рассеяния:

$$
f_{ \pm}(x, \lambda)=\phi_{0}( \pm x, \lambda) \pm \int_{x}^{ \pm \infty} K^{ \pm}(x, t) \phi_{0}( \pm t, \lambda) d t
$$

Ядра $K^{ \pm}(x, t)$ вещественны и удовлетворяют соотношениям

$$
K^{ \pm}(x, t)=O\left(\int_{(x+t) / 2}^{ \pm \infty}|q(s)| d s\right), \quad x+t \rightarrow \pm \infty, \quad K^{ \pm}(x, x)= \pm \frac{1}{2} \int_{x}^{ \pm \infty} q(s) d s .
$$

Согласно $(8),(9)$ решения $f_{ \pm}(x, \lambda)$ допускают аналитические продолжения в полуплоскость $\operatorname{Im} \lambda>0$. Кроме того, ввиду вещественности потенциала $q(x)$ и оценки $(9)$ на непрерывном спектре пары решений $\left\{f_{ \pm}(x, \lambda), \overline{f_{ \pm}(x, \lambda)}\right\}$ линейно независимы, поскольку их вронскиан $W\left\{f_{ \pm}(x, \lambda), \overline{f_{ \pm}(x, \lambda)}\right\}$ равен $\mp i \sqrt{2} e^{\pi \lambda / 4}$. Поэтому для вещественных значений $\lambda$ справедливы разложения

$$
\begin{aligned}
& f_{-}(x, \lambda)=a(\lambda) \overline{f_{+}(x, \lambda)}+b(\lambda) f_{+}(x, \lambda), \\
& f_{+}(x, \lambda)=a(\lambda) \overline{f_{-}(x, \lambda)}-\overline{b(\lambda)} f_{-}(x, \lambda),
\end{aligned}
$$

откуда следует, что матрица перехода имеет вид $T(\lambda)=\left(\frac{a(\lambda)}{b(\lambda)} \frac{b(\lambda)}{a(\lambda)}\right)$. Из соотношений (10), (11) следует, что

$$
|a(\lambda)|^{2}-|b(\lambda)|^{2}=1
$$


т. е. матрица перехода унимодулярна: $\operatorname{det} T(\lambda)=1$. Величины $r^{+}(\lambda)=b(\lambda) / a(\lambda)$ и $r^{-}(\lambda)=-\overline{b(\lambda)} / a(\lambda)$ назовем правым и левым коэффициентами отражения соответственно. Из формулы (10) имеем

$$
\begin{aligned}
& a(\lambda)=\frac{i}{\sqrt{2}} e^{-\pi \lambda / 4} W\left\{f_{+}(x, \lambda), f_{-}(x, \lambda)\right\}, \\
& b(\lambda)=-\frac{i}{\sqrt{2}} e^{-\pi \lambda / 4} W\left\{\overline{f_{+}(x, \lambda)}, f_{-}(x, \lambda)\right\} .
\end{aligned}
$$

Используя (8), (9), (13), найдем, что при $\lambda \rightarrow \infty$ справедливы соотношения

$$
a(\lambda)=a_{0}(\lambda)\left[1+O\left(\lambda^{-1 / 2}\right)\right], \quad b(\lambda)=b_{0}(\lambda)\left[1+O\left(\lambda^{-1 / 2}\right)\right] .
$$

Из (13) следует, что функция $a(\lambda)$ допускает аналитическое продолжение в верхнюю полуплоскость $\operatorname{Im} \lambda>0$ и непрерывна до вещественной оси. Кроме того, $a(\lambda)$ не имеет нулей. Действительно, на вещественной оси $a(\lambda)$ не обращается в нуль в силу условия нормировки (12). Предположим теперь, что $a\left(\lambda_{0}\right)=0, \operatorname{Im} \lambda_{0}>0$. Тогда из (13) получаем, что решения $f_{+}\left(x, \lambda_{0}\right)$ и $f_{-}\left(x, \lambda_{0}\right)$ линейно зависимы. Тогда формально самосопряженная задача (1) имела бы комплексное собственное значение, что невозможно.

Отметим, что аналогично формулам (7) имеют место следующие формулы разложения по собственным функциям непрерывного спектра уравнения (1):

$$
\frac{1}{\sqrt{2} \pi} \int_{-\infty}^{\infty} \operatorname{Re}\left\{\left[\overline{f_{ \pm}(x, \lambda)}+r^{ \pm}(\lambda) f_{ \pm}(x, \lambda)\right] f_{ \pm}(y, \lambda)\right\} \omega_{0}(\lambda) d \lambda=\delta(x-y) .
$$

Матрица перехода $T(\lambda)$ дает исчерпывающую информацию о поведении собственных функций непрерывного спектра задачи (1). Подчеркнем, что фактически вся информация о матрице $T(\lambda)$ содержится в одном из коэффициентов отражения. В самом деле, пусть, например, задан коэффициент $r^{+}(\lambda)$. Учитывая условие нормировки (12) точно так же, как и в работах [6], [7], получим

$$
a(z)=a_{0}(z) \exp \left\{-\frac{1}{2 \pi i} \int_{-\infty}^{\infty} \frac{\ln \left(1-\left|r^{+}(\lambda)\right|^{2}\right)-\ln \left(1-\left|r_{0}(\lambda)\right|^{2}\right)}{\lambda-z} d \lambda\right\}, \quad \operatorname{Im} z>0 .
$$

Коэффициенты $b(\lambda), r^{-}(\lambda)$ теперь могут быть построены на основании формул

$$
b(\lambda)=a(\lambda) r^{+}(\lambda), \quad r^{-}(\lambda)=-\overline{r^{+}(\lambda)} \frac{\overline{a(\lambda)}}{a(\lambda)} .
$$

В заключение этого раздела отметим, что из соотношений (12)-(14) вытекают основные свойства коэффициентов отражения. Перепишем их в виде условия.

УСловиЕ 1. Функиии $r^{ \pm}(\lambda)$ непрерывны на вещественной оси и удовлетворяют соотношениям

$$
\left|r^{ \pm}(\lambda)\right|<1, \quad r^{ \pm}(\lambda)-r_{0}(\lambda)=O\left(|\lambda|^{-1 / 2}\right) \cdot \begin{cases}e^{-\pi \lambda / 2}, & \lambda \rightarrow+\infty \\ 1, & \lambda \rightarrow-\infty\end{cases}
$$

Имеет место формула связи $r^{-}(\lambda) / r^{+}(\lambda)=-\overline{a(\lambda)} / a(\lambda)$, где $a(\lambda)$ определяется формулой (14). 


\section{4. ОБРАТНАЯ ЗАДАЧА РАССЕЯНИЯ}

Обратная задача рассеяния для уравнения (1) состоит в восстановлении потенциала $q(x)$ по одному из коэффициентов отражения. При решении обратной задачи важную роль играют основные интегральные уравнения Гельфанда-Левитана-Марченко.

Теорема 1. При каждом фиксированном х функции $K^{ \pm}(x, y)$, входящие в представления (8), удовлетворяют интегральным уравнениям

$$
F^{ \pm}(x, y)+K^{ \pm}(x, y) \pm \int_{x}^{ \pm \infty} K^{ \pm}(x, t) F^{ \pm}(t, y) d t=0, \quad \pm y> \pm x
$$

где

$$
F^{ \pm}(x, y)=\frac{1}{\sqrt{2} \pi} \operatorname{Re} \int_{-\infty}^{\infty}\left(r^{ \pm}(\lambda)-r_{0}(\lambda)\right) \phi_{0}( \pm x, \lambda) \phi_{0}( \pm y, \lambda) \omega_{0}(\lambda) d \lambda .
$$

ДоКАЗАТЕЛЬСТво. Для вывода уравнений (18) воспользуемся формулами разложения (7), (15). Для определенности рассмотрим случай со знаком плюс. Из известных свойств операторов преобразования (см., например, [7]) и из представлений (8) вытекает, что

$$
\phi_{0}(y, \lambda)=f_{+}(y, \lambda)+\int_{y}^{\infty} K(y, t) f_{+}(t, \lambda) d t
$$

где ядро $K(y, t)$ удовлетворяет соотношению, аналогичному (9). Тогда при $y>x$ из последней формулы с учетом (15) следует, что

$$
\begin{aligned}
& \frac{1}{\sqrt{2} \pi} \int_{-\infty}^{\infty} \operatorname{Re}\left\{\left[\overline{f_{+}(x, \lambda)}+r^{+}(\lambda) f_{+}(x, \lambda)\right] \phi_{0}(y, \lambda)\right\} \omega_{0}(\lambda) d \lambda= \\
& =\delta(x-y)+\int_{-\infty}^{y} K(y, t)\left(\frac { 1 } { \sqrt { 2 } \pi } \int _ { - \infty } ^ { \infty } \operatorname { R e } \left\{\left[\overline{f_{+}(x, \lambda)}+\right.\right.\right. \\
& \left.\left.\left.\quad+r^{+}(\lambda) f_{+}(x, \lambda)\right] f_{+}(t, \lambda)\right\} \omega_{0}(\lambda) d \lambda\right) d t= \\
& =\delta(x-y)+\int_{-\infty}^{y} K(y, t) \delta(x-t) d t=\delta(x-y)+K(y, x)=\delta(x-y) .
\end{aligned}
$$

С другой стороны, используя (7), (8), получаем

$$
\begin{aligned}
& \frac{1}{\sqrt{2} \pi} \int_{-\infty}^{\infty} \operatorname{Re}\left\{\left[\overline{f_{+}(x, \lambda)}+r^{+}(\lambda) f_{+}(x, \lambda)\right] \phi_{0}(y, \lambda)\right\} \omega_{0}(\lambda) d \lambda= \\
& =\frac{1}{\sqrt{2} \pi} \int_{-\infty}^{\infty} \operatorname{Re}\left\{\left[\overline{\phi_{0}(x, \lambda)}+r_{0}^{+}(\lambda) \phi_{0}(x, \lambda)\right] \phi_{0}(y, \lambda)\right\} \omega_{0}(\lambda) d \lambda+ \\
& \quad+\int_{x}^{\infty} K^{+}(x, t)\left\{\frac{1}{\sqrt{2} \pi} \int_{-\infty}^{\infty} \operatorname{Re}\left\{\left[\overline{\phi_{0}(t, \lambda)}+r_{0}^{+}(\lambda) \phi_{0}(t, \lambda)\right] \phi_{0}(y, \lambda)\right\} \omega_{0}(\lambda) d \lambda\right\} d t+ \\
& \quad+\frac{1}{\sqrt{2} \pi} \int_{-\infty}^{\infty} \operatorname{Re}\left\{\left[r^{+}(\lambda)-r_{0}^{+}(\lambda)\right] \phi_{0}(x, \lambda) \phi_{0}(y, \lambda)\right\} \omega_{0}(\lambda) d \lambda+ \\
& \quad+\int_{x}^{\infty} K^{+}(x, t)\left\{\frac{1}{\sqrt{2} \pi} \int_{-\infty}^{\infty} \operatorname{Re}\left\{\left[r^{+}(\lambda)-r_{0}^{+}(\lambda)\right] \phi_{0}(t, \lambda) \phi_{0}(y, \lambda)\right\} \omega_{0}(\lambda) d \lambda\right\} d t=
\end{aligned}
$$




$$
\begin{aligned}
= & \delta(x-y)+\int_{x}^{\infty} K^{+}(x, t) \delta(t-y) d t+ \\
& +\frac{1}{\sqrt{2} \pi} \int_{-\infty}^{\infty} \operatorname{Re}\left\{\left[r^{+}(\lambda)-r_{0}^{+}(\lambda)\right] \phi_{0}(x, \lambda) \phi_{0}(y, \lambda)\right\} \omega_{0}(\lambda) d \lambda+ \\
& +\int_{x}^{\infty} K^{+}(x, t)\left\{\frac{1}{\sqrt{2} \pi} \int_{-\infty}^{\infty} \operatorname{Re}\left\{\left[r^{+}(\lambda)-r_{0}^{+}(\lambda)\right] \phi_{0}(t, \lambda) \phi_{0}(y, \lambda)\right\} \omega_{0}(\lambda) d \lambda\right\} d t= \\
= & \delta(x-y)+K^{+}(x, y)+F^{+}(x, y)+\int_{x}^{\infty} K^{+}(x, t) F^{+}(t, y) d t .
\end{aligned}
$$

Сопоставляя это равенство с равенством (20), получаем (17). Теорема доказана.

Основные уравнения позволяют решить обратную задачу следующим образом. При помощи основных уравнений (18), как и в работах [10], [21], устанавливается следующее свойство функций рассеяния $F^{ \pm}(x, y)$.

УСловиЕ 2. Функиии $F^{ \pm}(x, y)$, определенные формулами (19), непрерывно дибферениируемы и удовлетворяют соотношениям

$$
\begin{aligned}
\left|F^{ \pm}(x, y)\right| \leqslant C_{ \pm}(a), \quad \pm x \geqslant a, \quad \pm y \geqslant a, \quad\left|\int_{a}^{ \pm \infty} \sup _{ \pm x>a}\right| F^{ \pm}(x, t)|d t|<\infty \\
\lim _{N \rightarrow \pm \infty}\left|\int_{N}^{ \pm \infty} \sup _{ \pm(x-a) \geqslant 0}\right| F^{ \pm}(x, y)|d y|=0 \\
\left(1+\left|x_{1}\right|\right) \sup _{ \pm t \geqslant 0}\left|\int_{x_{1}}^{\infty}\right| F_{x_{i}}^{ \pm}\left(x_{1}+t, x_{2}\right)\left|d x_{2}\right| \leqslant C_{i}^{ \pm}(a), \quad \pm\left(x_{1}-a\right) \geqslant 0 \\
\left|\int_{a}^{ \pm \infty}\left(1+x^{4}\right)\right| \frac{d}{d x} F^{ \pm}(x, x)|d x|<C_{0}^{ \pm}(a), \\
\lim _{h \rightarrow 0} \sup _{ \pm(z-x) \geqslant 0}\left|\int_{x}^{ \pm \infty}\right| F^{ \pm}(z, y+h)-F^{ \pm}(z, y)|d y|=0 .
\end{aligned}
$$

ТЕОрема 2. Пусть выполняются условия 1, 2. Тогда при каждом фиксированном $x$ уравнения (18) имеют единственные решения $K^{ \pm}(x, \cdot) \in L_{p}(x, \pm \infty), p=1,2$.

ДокАЗАТЕльство. Из условия 2 следует, что уравнения (18) порождаются вполне непрерывными операторами. Поэтому согласно альтернативе Фредгольма искомое решение уравнения (18) существует и единственно в пространстве $L_{p}(x, \infty), p=1,2$, если у однородного уравнения отсутствуют нетривиальные решения из $L_{p}(x, \infty)$, $p=1,2$.

Не нарушая общности, будем рассматривать случай со знаком плюс. Пусть при каком-либо $x$ однородное уравнение

$$
h(y)+\int_{x}^{\infty} F^{+}(y, t) h(t) d t=0, \quad \pm y> \pm x,
$$

имеет нетривиальное решение из $L_{2}(x, \infty)$. В силу вещественности ядра $F^{+}(x, y)$ можно считать, что $h(y)$ принимает вещественные значения. Тогда верно равенство

$$
\int_{x}^{\infty}|h(y)|^{2} d y+\int_{x}^{\infty} \int_{x}^{\infty} F^{+}(y, t) h(t) h(y) d t d y=0 .
$$


Подставляя в последнее равенство функции $F^{+}(x, y)$ из $(19)$, получаем

$$
\begin{aligned}
& \int_{x}^{\infty}|h(y)|^{2} d y+ \\
& +\frac{1}{2 \sqrt{2} \pi} \int_{-\infty}^{\infty}\left(r^{+}(\lambda)-r_{0}^{+}(\lambda)\right)\left[\int_{x}^{\infty} \phi_{0}(t, \lambda) h(t) d t\right]\left[\int_{x}^{\infty} \phi_{0}(y, \lambda) h(y) d y\right] \omega_{0}(\lambda) d \lambda+ \\
& +\frac{1}{2 \sqrt{2} \pi} \int_{-\infty}^{\infty}\left(\overline{r^{+}(\lambda)}-\overline{r_{0}^{+}(\lambda)}\right)\left[\int_{x}^{\infty} \overline{\phi_{0}(t, \lambda)} h(t) d t\right]\left(\int_{x}^{\infty} \overline{\phi_{0}(y, \lambda)} h(y) d y\right] \omega_{0}(\lambda) d \lambda=0
\end{aligned}
$$

Перепишем последнее равенство в виде

$$
\begin{aligned}
& \int_{x}^{\infty}|h(y)|^{2} d y+ \\
& +\frac{1}{2 \sqrt{2} \pi} \int_{-\infty}^{\infty} r^{+}(\lambda)\left[\int_{x}^{\infty} \phi_{0}(t, \lambda) h(t) d t\right]\left[\int_{x}^{\infty} \phi_{0}(y, \lambda) h(y) d y\right] \omega_{0}(\lambda) d \lambda+ \\
& +\frac{1}{2 \sqrt{2} \pi} \int_{-\infty}^{\infty} \overline{r^{+}(\lambda)}\left[\int_{x}^{\infty} \overline{\phi_{0}(t, \lambda)} h(t) d t\right]\left[\int_{x}^{\infty} \overline{\phi_{0}(y, \lambda)} h(y) d y\right] \omega_{0}(\lambda) d \lambda- \\
& -\frac{1}{2 \sqrt{2} \pi} \int_{-\infty}^{\infty} r_{0}^{+}(\lambda)\left[\int_{x}^{\infty} \phi_{0}(t, \lambda) h(t) d t\right]\left[\int_{x}^{\infty} \phi_{0}(y, \lambda) h(y) d y\right] \omega_{0}(\lambda) d \lambda- \\
& -\frac{1}{2 \sqrt{2} \pi} \int_{-\infty}^{\infty} \overline{r_{0}^{+}(\lambda)}\left[\int_{x}^{\infty} \overline{\phi_{0}(t, \lambda)} h(t) d t\right]\left[\int_{x}^{\infty} \overline{\phi_{0}(y, \lambda)} h(y) d y\right] \omega_{0}(\lambda) d \lambda=0 .
\end{aligned}
$$

С другой стороны, в силу формулы разложения (7) имеем

$$
\begin{aligned}
\int_{x}^{\infty}|h(y)|^{2} d y=\int_{-\infty}^{\infty} & {\left[\frac{1}{\sqrt{2} \pi} \int_{x}^{\infty} h(t) \phi_{0}(t, \lambda) d t\right] \times } \\
\times & {\left[\int_{x}^{\infty} h(y)\left(\overline{\phi_{0}(y, \lambda)}+r_{0}^{+}(\lambda) \phi_{0}(y, \lambda)\right) d y\right] \omega_{0}(\lambda) d \lambda } \\
\int_{x}^{\infty}|h(y)|^{2} d y=\int_{-\infty}^{\infty} & {\left[\frac{1}{\sqrt{2} \pi} \int_{x}^{\infty} h(t) \overline{\phi_{0}(t, \lambda)} d t\right] \times } \\
\times & {\left[\int_{x}^{\infty} h(y)\left(\overline{\phi_{0}(y, \lambda)}+\overline{r_{0}^{+}(\lambda)} \overline{\phi_{0}(y, \lambda)}\right) d y\right] \omega_{0}(\lambda) d \lambda }
\end{aligned}
$$

Отсюда следует, что

$$
\begin{aligned}
\int_{x}^{\infty}|h(y)|^{2} d y & -\frac{1}{2 \sqrt{2} \pi} \int_{-\infty}^{\infty} r_{0}^{+}(\lambda)\left[\int_{x}^{\infty} h(y) \phi_{0}(y, \lambda) d y\right]^{2} \omega_{0}(\lambda) d \lambda- \\
& -\frac{1}{2 \sqrt{2} \pi} \int_{-\infty}^{\infty} \overline{r_{0}^{+}(\lambda)}\left[\int_{x}^{\infty} h(y) \overline{\phi_{0}(y, \lambda)} d y\right]^{2} \omega_{0}(\lambda) d \lambda= \\
= & \frac{1}{\sqrt{2} \pi} \int_{-\infty}^{\infty}\left|\int_{x}^{\infty} h(y) \phi_{0}(y, \lambda) d y\right|^{2} d \lambda .
\end{aligned}
$$

Учитывая эти формулы в равенстве (22) и полагая

$$
H(\lambda)=\int_{x}^{\infty} h(y) \phi_{0}(y, \lambda) d y
$$


получаем

$$
\begin{gathered}
\int_{-\infty}^{\infty}|H(\lambda)|^{2} \omega_{0}(\lambda) d \lambda+\frac{1}{2} \int_{-\infty}^{\infty} r^{+}(\lambda) H^{2}(\lambda) \omega_{0}(\lambda) d \lambda+ \\
+\frac{1}{2} \int_{-\infty}^{\infty} \overline{r^{+}(\lambda)} \overline{H^{2}(\lambda)} \omega_{0}(\lambda) d \lambda=0
\end{gathered}
$$

С учетом последнего соотношения заключаем, что

$$
\int_{-\infty}^{\infty}\left(1-\left|r^{+}(\lambda)\right|\right)|H(\lambda)|^{2} \omega_{0}(\lambda) d \lambda \leqslant 0
$$

откуда следует, что

$$
H(\lambda)=\int_{x}^{\infty} h(y) \phi_{0}(y, \lambda) d y \equiv 0,
$$

так как $1-\left|r^{+}(\lambda)\right|>0$ и $\omega_{0}(\lambda)=e^{-\pi \lambda / 4}>0$ при всех действительных значениях $\lambda$. Из последнего тождества вытекает, что $h(y) \equiv 0$.

Таким образом, однородное уравнение (21) имеет лишь тривиальное решение из $L_{2}(x, \infty)$. Единственность решения в $L_{1}(x, \infty)$ вытекает из того, что любое решение $h(y)$ уравнения $(21)$ из $L_{1}(x, \infty)$ принадлежит $L_{2}(x, \infty)$. Доказательство последнего утверждения точно такое же, как в работе [21]. Теорема доказана.

В заключение отметим, что установленные выше условия 1, 2 также являются достаточными, чтобы по правому коэффициенту отражения можно было однозначно восстановить потенциал $q(x)$ из класса

$$
\int_{-\infty}^{\infty}\left(1+|x|^{4}\right)|p(x)| d x<\infty .
$$

В самом деле, пусть дан правый коэффициент отражения $r^{+}(\lambda)$. При помощи формул $(15),(16)$ найдем функции $a(\lambda), r^{-}(\lambda)$. По формулам (19) определим функции $F^{ \pm}(x, y)$. При условиях 1,2 основные уравнения (18) имеют единственные решения $K^{ \pm}(x, y)$. Тогда, как и в работах [6], [7], [9], [10], можно доказать, что определенные формулами $(8)$ функции $f_{ \pm}(x, \lambda)$ являются решениями уравнений

$$
-f_{ \pm}^{\prime \prime}(x, \lambda)+\left[-x^{2}+q^{ \pm}(x)\right] f_{ \pm}(x, \lambda)=\lambda f_{ \pm}(x, \lambda),
$$

где потенциалы $q^{ \pm}(x)$ определяются формулами

$$
q^{ \pm}(x)=\mp 2 \frac{d K^{ \pm}(x, x)}{d x}
$$

и удовлетворяют при каждом $a$ неравенствам

$$
\left|\int_{a}^{ \pm \infty}\left(1+|x|^{4}\right)\right| q^{ \pm}(x)|d x|<\infty .
$$

Далее, аналогично [6], [7], [21] доказывается, что при действительных значениях $\lambda$ функции $f_{ \pm}(x, \lambda)$ связаны равенствами

$$
\begin{aligned}
& \frac{1}{a(\lambda)} f_{-}(x, \lambda)=\overline{f_{+}(x, \lambda)}+r^{+}(\lambda) f_{+}(x, \lambda), \\
& \frac{1}{a(\lambda)} f_{+}(x, \lambda)=\overline{f_{-}(x, \lambda)}+r^{-}(\lambda) f_{-}(x, \lambda),
\end{aligned}
$$

из которых вытекает равенство $q^{+}(x)=q^{-}(x)$. 
Благодарности. Авторы выражают благодарность рецензенту за полезные замечания, которые способствовали улучшению содержания работы.

\section{Список литературы}

[1] Ф. А. Березин, М. А. Шубин, Уравнение Шредингера, Изд-во Моск. ун-та, М., 1983.

[2] H. P. McKean, E. Trubowitz, "The spectral class of the quantum-mechanical harmonic oscillator", Commun. Math. Phys., 82:4 (1982), 471-495.

[3] Б. М. Левтан, "Об операторах Штурма-Лиувилля на всей прямой с одинаковым дискретным спектром", Матем. сб., 132(174):1 (1987), 73-103.

[4] D. Chelkak, P. Kargaev, E. Korotyaev, "Inverse problem for harmonic oscillator perturbed by potential, characterization", Commun. Math. Phys., 249:1 (2004), 133-196.

[5] Л. Д. Фаддеев, “Обратная задача квантовой теории рассеяния”, УМH, 14:4 (1959), $57-119$.

[6] Л.Д. Фаддеев, "Свойства $S$-матрицы одномерного уравнения Шредингера", $T p$. МИАН СССР, 73 (1964), 314-326.

[7] В. А. Марченко, Операторы Штурма-Лиувилля и их приложения, Наукова думка, Киев, 1977.

[8] М. Г. Гасымов, Б. А. Мустафаев, “Обратная задача рассеяния для ангармонического уравнения на полуоси", Докл. АН СССР, 228:1 (1976), 11-14.

[9] Y. Li, "One special inverse problem of the second order differential equation on the whole real axis", Chinese Ann. Math., 2:2 (1981), 147-155.

[10] А. П. Качалов, Я. В. Курылев, “Метод операторов преобразования в обратной задаче рассеяния. Одномерный штарк-эффект", Зап. научн. сем. ЛОМИ, 179 (1989), 73-87.

[11] П. П. Кулиш, "Обратная задача рассеяния для уравнения Шредингера на оси”, Матем. заметки, 4:6 (1968), 677-684.

[12] Y. Liu, "Scattering and spectral theory for Stark Hamiltonians in one dimension", Math. Scand., 72:2 (1993), 265-297.

[13] Е.А. Кузнецов, А. В. Михайлов, “Устойчивость стационарных волн в нелинейных средах со слабой дисперсией”, ЖЭТФ, 67:5 (1974), 1717-1727.

[14] А. М. Савчук, А. А. Шкаликов, "Спектральные свойства комплексного оператора Эйри на полуоси", Функи. анализ и его прил., 51:1 (2017), 82-98.

[15] B. S. Mityagin, P. Siegl, "Root system of singular perturbations of the harmonic oscillator type operators", Lett. Math. Phys., 106:2 (2016), 147-167.

[16] E. L. Korotyaev, "Resonances for 1d Stark operators", J. Spectr. Theory, 7:3 (2017), 699-732, arXiv: 1703.10820.

[17] Е. Л. Коротяев, "О рассеянии во внешнем однородном периодическом по времени магнитном поле", Матем. сб., 180:4 (1989), 491-512.

[18] М. Абрамович, И. Стиган (ред.), Справочник по специалъным функииям с формулами, графиками и математическими таблицами, Наука, М., 1979.

[19] Г. Бейтмен, А. Эрдейи, Высшие трансцендентные функиии, т. 2: Функиии Бесселя, функиии параболического иилиндра, ортогональные многочлены, Наука, М., 1974.

[20] Э. Ч. Титчмарш, Разложения по собственным функциям, связанные с дифференциальными уравнениями второго порядка, т. 1, ИЛ, М., 1960.

[21] Н.Е. Фирсова, "Прямая и обратная задачи рассеяния для возмущенного оператора Хилла", Матем. сб., 130:3 (1986), 349-385.

Поступила в редакцию 20.06.2017, после доработки 13.08.2017 